\title{
Realization of a Method to Control Electrical Appliances through Web Interface
}

\author{
Siraj Ahmed ${ }^{1}$, Mir Mohammed Ali ${ }^{2}$, Mohammed Abdul Sami Siddiqui ${ }^{3}$ \\ ${ }^{1}$ Maturi Venkata Subba Rao Engineering College, Department of Electrical and Electronics, Nadergul, Hyderabad, Telangana, India \\ ${ }^{2}$ Mahaveer Institute Of Science and Technology, Department of Electronics and Communication, Bandlaguda, Hyderabad ,Telangana, India \\ ${ }^{3}$ Gokaraju Ranga Ranju Institute of Technology, Department of Electrical and Electronics, Bachupally, Hyderabad, Telangana ,India
}

\begin{abstract}
The technology has been advancing in the area of controlling high power equipment using low power flexible techniques such as Infrared Remote, Voice, Bluetooth, Zigbee, so on. This research paper elucidates the practical realization of one such technique -"web interface " to switch $\mathrm{ON} / \mathrm{OFF}$ any electrical equipment in industry/home from any part of the world through a website. Though many methods are already proposed, this project has come up with a unique hardware design and software code which allows the user to control up to a maximum of 23 electrical loads with just one module, making it highly efficient and economical.
\end{abstract}

Keywords: IoT, website, electrical appliances, economical, Raspberry Pi.

\section{Introduction}

The advancements in the technology in the past few decades has resulted in the automation of almost every industry, power plants, oil and mining plant. The safety and the flexibility employed in the use of computers to control high power equipment resulted in a lot of research in embedding different disciplines of electrical, mechanical and mining with computer sciences. With the advent of microcomputers, techniques such as use of Infrared, Bluetooth, Zigbee, Voice, were used to switch ON/OFF high power electrical equipment .However they all require the user to be within a specified range of the equipment. Furthermore the techniques of missed call and SMS which are not limited by range of operation but demands an additional cell phone and sim card to be attached to the micro controller interfaced to the equipment, also is highly erroneous since the random phone calls and SMS from people, advertisements, customer care might trigger the output of microcontroller. Additionally, the technique of "web interface" to remotely switch ON/OFF an electrical device (in home or an industry) has been employed using a microcontroller. However it requires an Ethernet port to be attached to micro-controller decreasing the overall current output rating. Thereby limiting the number of loads that can be controlled and enhancing the cost if one desire to increase the number of loads.

This project works towards the use of a microprocessor Raspberry $P i$ instead of a microcontroller. The inbuilt Ethernet port on the Raspberry Pi and availability of 23 GP10 pins has enabled to design a practical circuit without add on peripherals. Thereby resulting in an economical and efficient module. Furthermore a unique method has been proposed and successfully implemented to interface the web and the microprocessor. It allows the user to open a website and switch ON/OFF the desired equipment at his house say a Bulb, or Fan, AC, Water Pump etc from any part of the world.

\section{Problem Statements}

The following are the major six problem statements the entire research has been broken down into:

1)Design a controllable low power switch which makes or breaks a high power circuit. (discussed in section 3.2)

2)Interface the low power switch to the Microprocessor pin. . (discussed in section 3.2)

3) Code the processor to output HIGH or LOW at the pin which feeds the low power switch.

4) Test the proper working of hardware interface (of Processor, low power switch and high power circuit ) by manually executing the files to control pin output.

5)Create a dynamic website with a personal domain registered over the internet.(discussed in section 3.3.1)

6)Interface the web server to the microprocessor.(discussed in section 3.3.2)

\section{Method and Realization}

\subsection{Overview}

The figure-1 describes briefly the procedure followed to switch ON/OFF an electrical device at your house/industry from any part of the world using a smart phone, laptop or any internet enabled device. The user has to search the URL of website from any electronic gadget using a web browser and after successful authentication the user is directed to a page which display the list of appliances installed in the house or factory. The user can then select to switch $\mathrm{ON}$ or switch OFF a particular device say a Bulb from the list and the same is updated into the MySQL database. The microprocessor now reflects the changes in the database and executes a file to output a high or low at one of its pin where that desired appliance $-B u l b$ is interfaced. The pin of the micro-processor which outputs a high $(3.3 \mathrm{v})$ or low $(0 \mathrm{v})$ depending on the execution of a file, is interfaced to the high power appliance (bulb) using a switching circuitry which will be discussed in the section 3.2(hardware implementation). 


\section{International Journal of Science and Research (IJSR) \\ ISSN (Online): 2319-7064 \\ Index Copernicus Value (2013): 6.14 | Impact Factor (2014): 5.611}

In the nutshell the execution of a file to output a high/low at the pin is governed by the changes in the database of the webserver which reflects the need of a user to either switch $\mathrm{ON}$ or switch OFF a particular equipment. The algorithm followed to code at the web server has been explained in section 3.3.1

\subsection{Hardware Realization:}

This section addresses the problem statements 1 and 2 from the section 2.0.The hardware implementation requires the processor Raspberry Pi2, Low power switching circuitry using opto coupler \& relay and the appliance which is to be controlled such as C.F.L, Bulb and Fan etc. The software schematics is as shown in figure 2. For sake of simplicity circuit for only three pins are shown, it is to be noted that all the 23 pins has the same interface circuit

As Raspberry pi 2 comprises 23 General Purpose Input Output (GPIO) pins which can be utilize to perform input output operations, these pins provide the voltage in the range $0 \mathrm{~V}$ to $3.3 \mathrm{~V}$ at each pin and exceed in currents and voltages leads to damage the Raspberry pi, to overcome this problem a low power switching circuitry is designed using Opto coupler, MOSFET and Relays.

A HIGH output (of $3.3 \mathrm{v}$ ) at GPIO, forward biases the internal LED of opto-coupler which in turn saturates the transistor coupled optically to the LED, eventually a threshold voltage applied to the MOSFET allows the excitation of relay coil. Upon excitation, the high power circuit consisting the appliance (Bulb) drives the power from household phase. Similarly, LOW output (of 0v) at GPIO, deactivates the opto coupler. Since the gate to source voltage is now zero, the MOSFET is derived from saturation to cutoff region de-energizing the relay coil. Following which the high power circuit gets open circuited.

Any fluctuation in the high power circuit due to damage in relay or MOSFET does not get reflected on the raspberry pi because of the use of opto coupler. Further a resistor R1 in series has connected with the opto coupler to limit the current from the pin to a safer value.

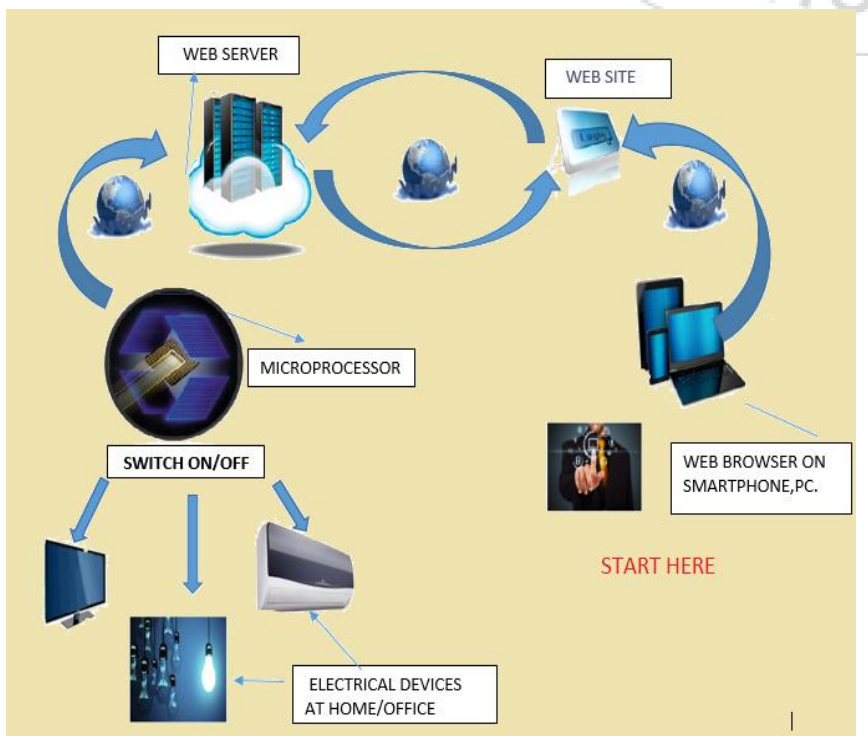

Figure 1: Overview of the Problem Statements

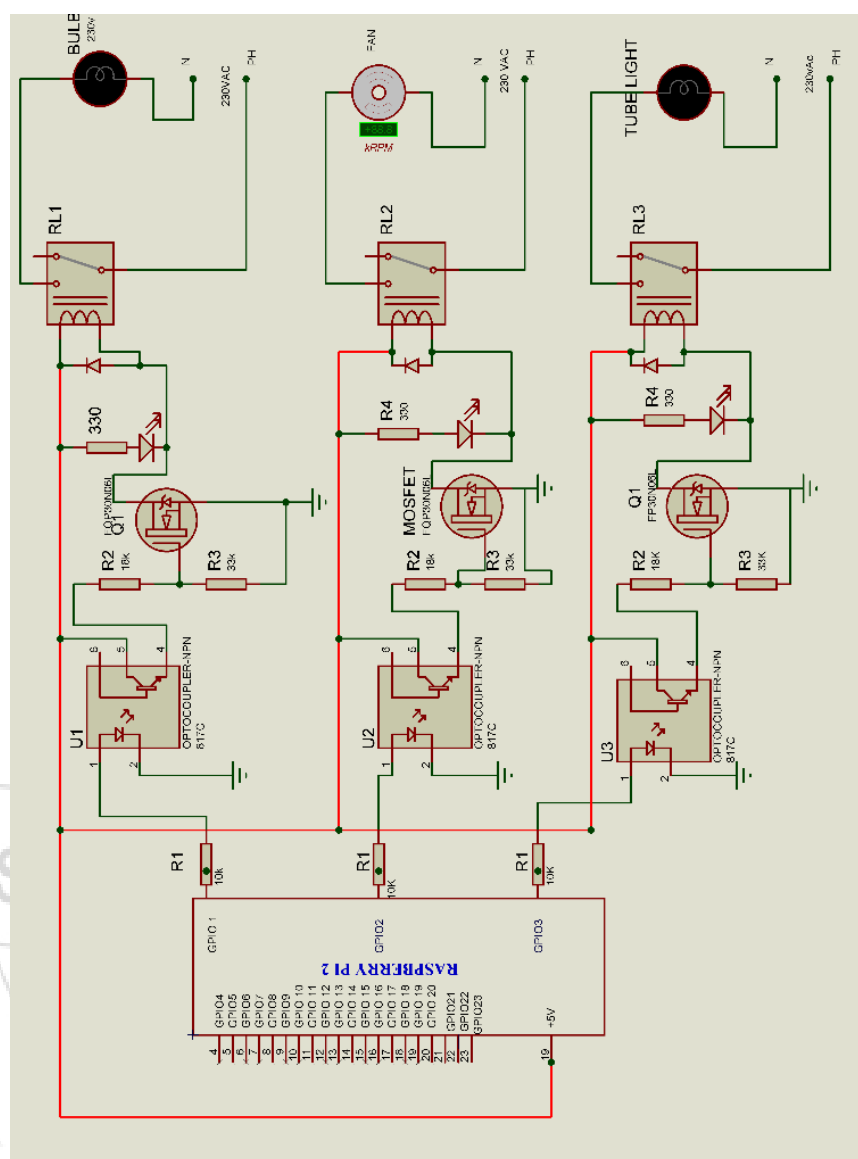

Figure 2: Hardware Implementation

\subsection{Software Implementation:}

The implementation of the algorithm to interface the microprocessor pins and the website can be divided into two parts. These sections deal with the problem statements 5 and 6 mentioned in section 2.0 Problem Statements.

1) Server Side Algorithm.

2) Processor Side Algorithm.

\subsubsection{Server Side Algorithm}

The user need to have a valid public domain registered with a web hosting service which provides complete access to PHP Scripting,MySQL Database, SSH support. The figure-3 shows the method to be followed for coding at the server side

1) The user creates a webpage consisting of an HTML/CSS form tag $<$ Select $>$ with two option values as ON and OFF.

2) The user has to then create database using SQL/MySQL which can hold a character value.

3)A code scripted in PHP has to be then written to link the database to the HTML form which is explained below in point 4.

4) Whenever the user wants to switch $\mathrm{ON}$ a device, he simply selects the ON option and press the submit button. This results in the updating a value "ON" in this database. If the existing value is "OFF" in the database, it is updated to "ON "If the existing value and the new value is same then it does not make any change and returns the status of the device. Similarly the OFF option can also be interpreted from the figure-3. 


\section{International Journal of Science and Research (IJSR) \\ ISSN (Online): 2319-7064 \\ Index Copernicus Value (2013): 6.14 | Impact Factor (2014): 5.611}

The following are the names of the files to be written in the public html folder of your account. The code for each file has been provided in the section 3.3.3.

1) Default.php 2)Proceed.php 3)link_db.php

Note:-

For the sake of simplicity code for only one pin has been given. For multiple entries up to 23 electrical devices the same code with different variables can be written in the files Proceed.php and Link_db.php.

\subsubsection{Processor_Side Algorithm}

After successful installation of operating system (Raspbian) In the Raspberry Pi open the command terminal and follow the procedure below:-

1)Create two files of python code to access a single GPI0 pin. One file TION.py to output a HIGH voltage at the pin and TIOFF.py to output low voltage at the pin (T1ON.PY, T10FF.PY files are coded in section 3.3.3).

2)A PHP script to link the terminal of RPI and the web database has to be written, the algorithm depicted in the figure 4 and explained as :

The microprocessor keeps checking the value from the database for every specified time interval, and executes python files accordingly. for instance if the value in the web database is $\mathrm{ON}$, the processor checks the previous status of pin and if LOW then a file to output HIGH voltage to the pin is executed otherwise ignores execution since the previous status is already HIGH . Similarly the OFF condition can be interpreted from the figure 4 .

3) Save all the files in a particular folder and note down the path.

4)Execute the command /usr/bin/php path/file.php $>/$ dev/null \&.

The following are the list of files to be saved in the RPI. The codes for the same are provided and in the section 3.3.3. For the sake of simplicity code for 1 pin has been shown.

1) T1ON.py 2)T1OFF.py 3) file.php 4) con.php

Note: For multiple loads, each pin must have two files of python code i.e. (T2ON.py, T2OFF.py) and so on, also make the changes in file.php by just copying the same code for different pins with changed variables.

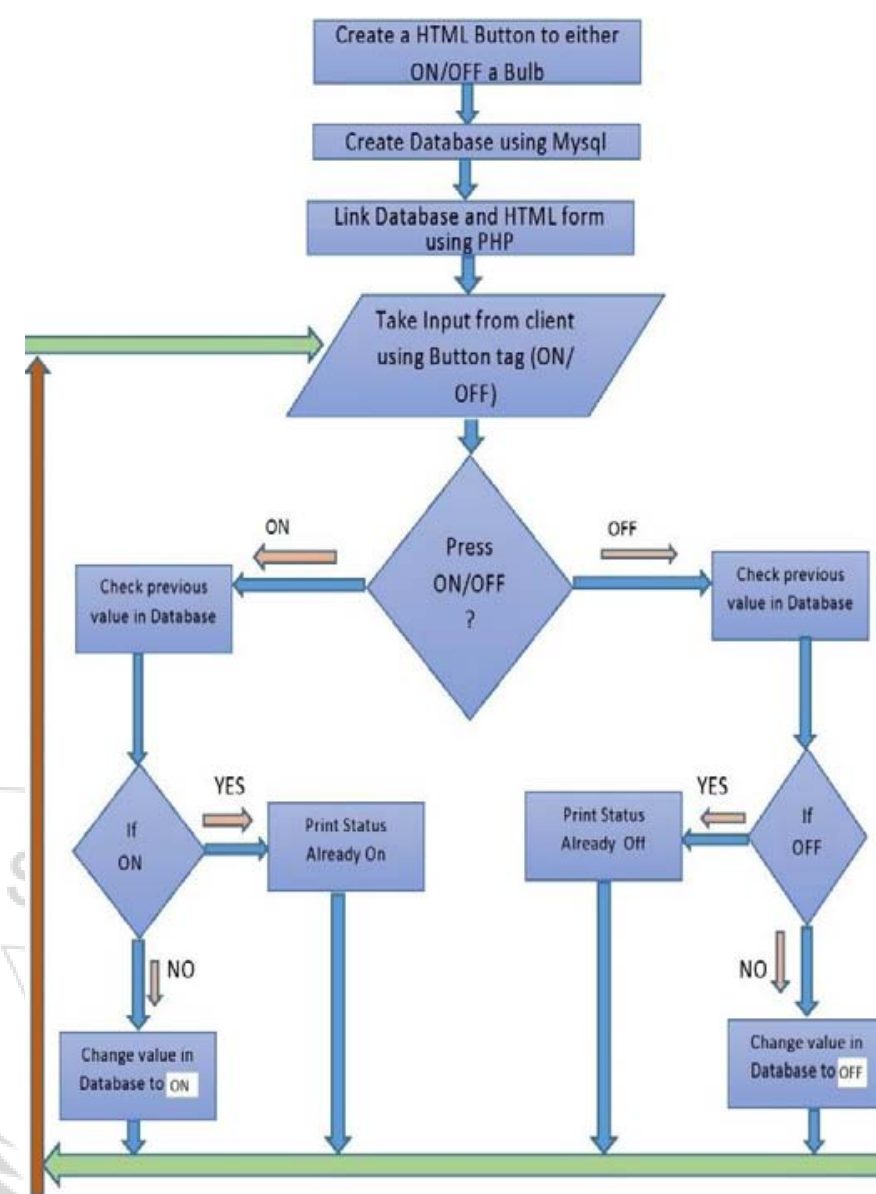

Figure 3: Web Server Side Algorithm

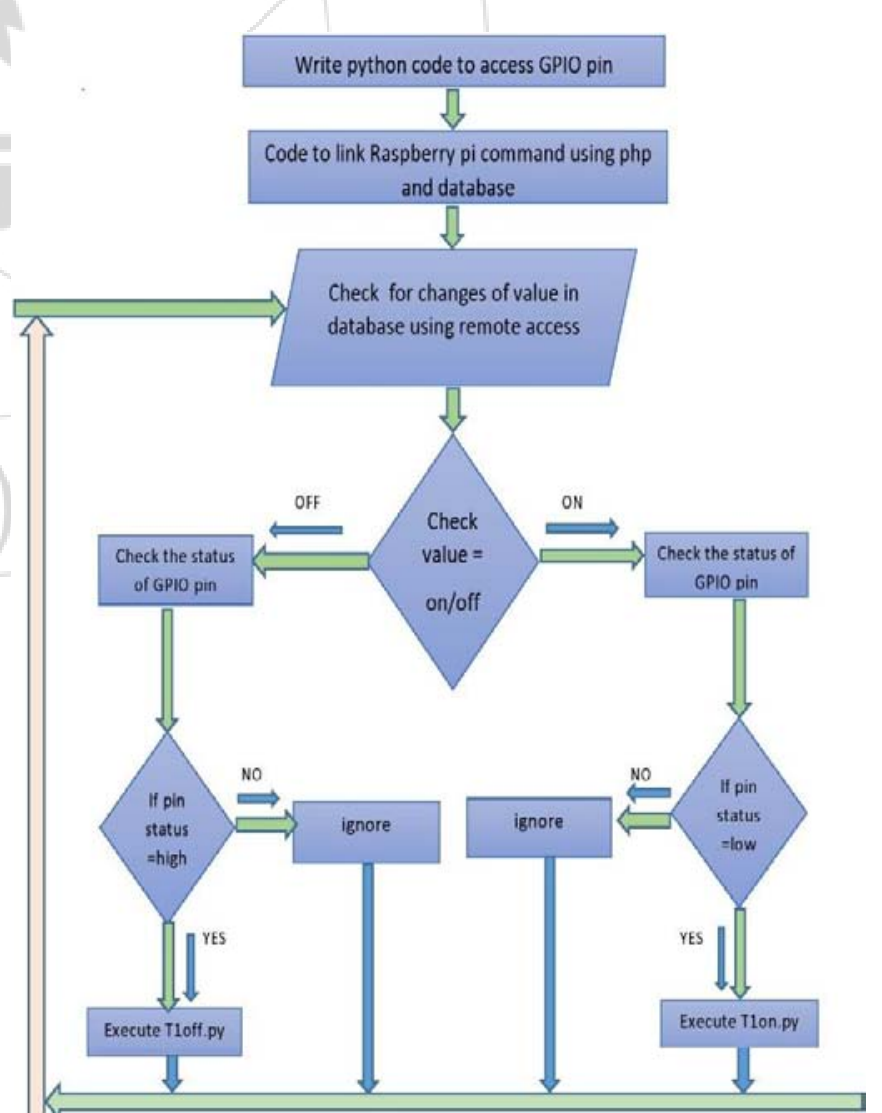

Figure 4: Processor Side Algorithm 


\section{International Journal of Science and Research (IJSR)}

ISSN (Online): 2319-7064

Index Copernicus Value (2013): 6.14 | Impact Factor (2014): 5.611

\subsubsection{CODES}

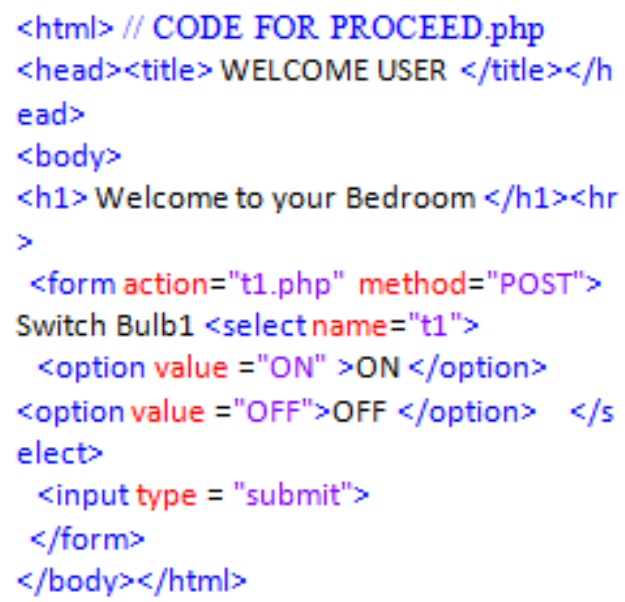

Code 1: Proceed.php

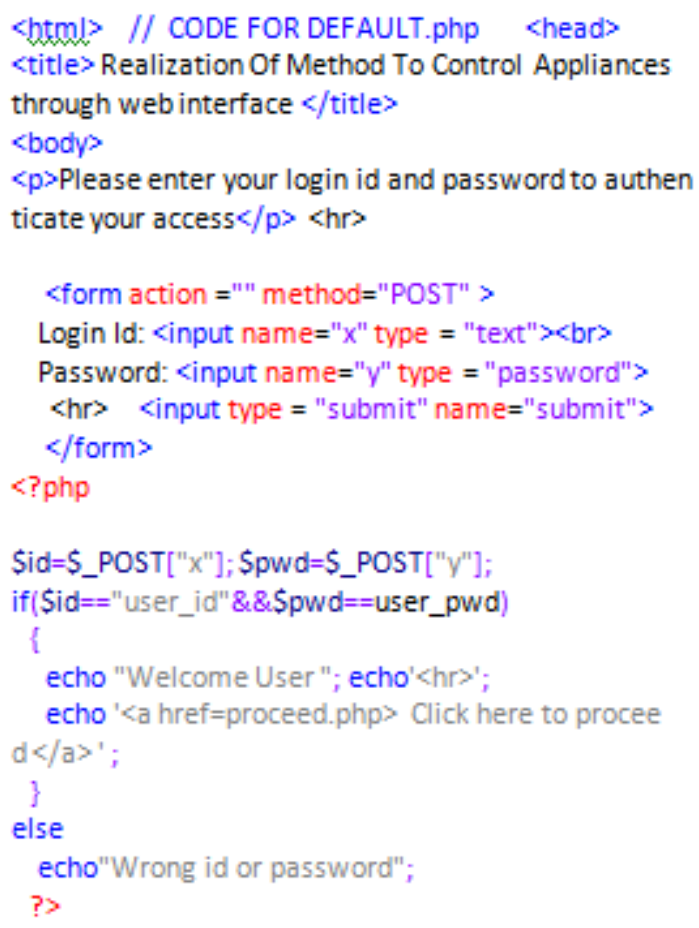

Code 2: Default.php <html> /CODEFORLINK_DB.php <head >

$<$ title $>$ Linking database and HTML form $</$ title $></$ hea d)

<bodys

$<$ ?php

$\$ c o n=m y s q \mid$ connect("Database_Hostname","Usema me","password") or die(mysql_error()):

mysql_select_db("database_name",\$con);

\$name $=\$ \_P O S T[" t 1 "]$;

if $($ \$name=="ON")

\{

mysql_query("update test set $\mathrm{t} 1=$ '1'") $^{\prime}$ or die(mysql_ error());

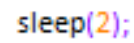

Code 3: Link_db.php

$/ /$ Code to output high voltage at pin import RPi.GPIO as GPIO GPIQ setwarnings (false) GPIO.setmode(GPIO.BOARD) GPIO.setup( PIN_NO, GPIO.OUT) GPIO.output(PIN NO,1)

\section{Code 4: T1ON.PY}

// Code to output Low voltage at pin
import RPi.GPIO as GPIO
GPIQ setwarnings(false)
GPIO.setmode(GPIO.BOARD)
GPIO.setup(PIN_NO,GPIO.OUT)
GPIO.output(PIN_NO,0)

Code 5: T1OFF.PY

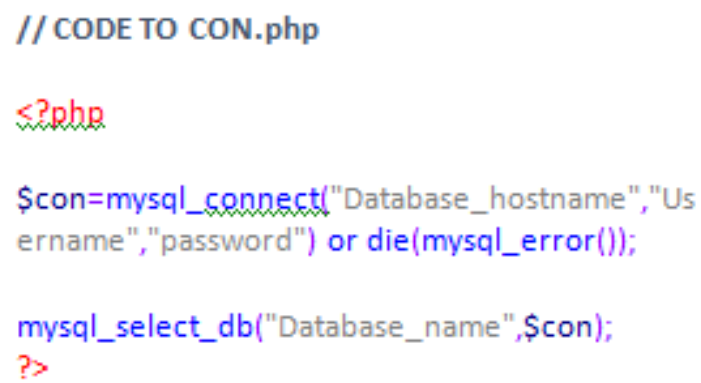

Code 6: con.php 


\section{International Journal of Science and Research (IJSR)}

ISSN (Online): 2319-7064

Index Copernicus Value (2013): 6.14 | Impact Factor (2014): 5.611

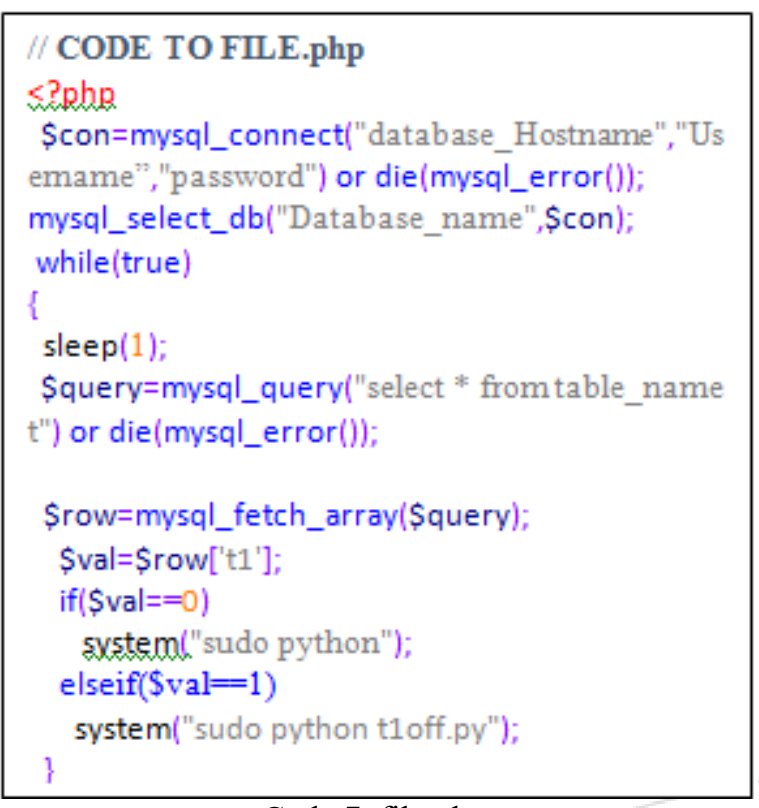

Code 7: file.php

\subsection{Experimental Results}

The implementation of both server side and processor side scripting results in the successful interface of the web database to the microprocessor. Now any person can search the URL of the website from his phone or PC and upon authentication has the access to the appliance.

The practical test results depicted from figure 5 (a) to figure 5(d) confirms the working of the hardware and software realization and the viability of the method proposed to switch $\mathrm{ON} / \mathrm{OFF}$ any electrical equipment at home/industry using a website no matter where the user is.

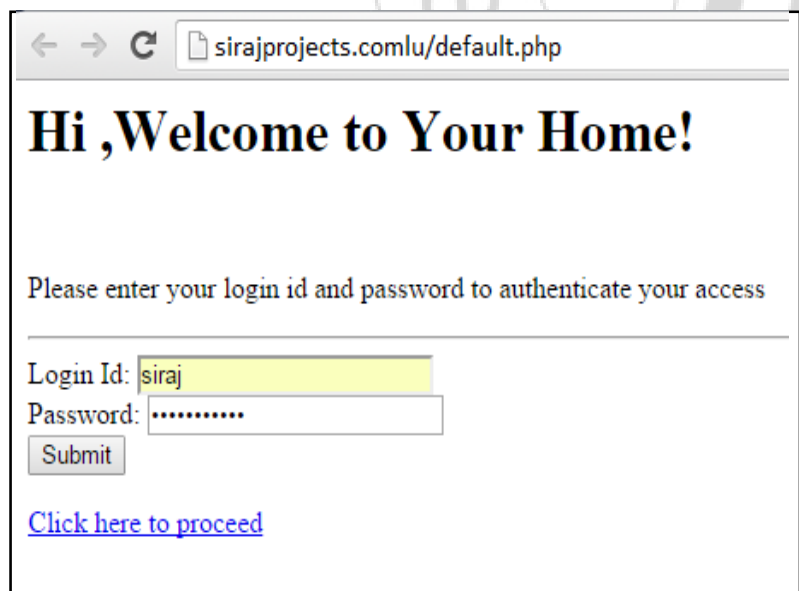

Figure 5(a): Search URL and authenticate.

\section{$\epsilon \rightarrow \mathrm{C}$ Dsirajprojects.comlu/proceed.php \\ Welcome to your Bedroom}

\section{Switch BULB 1 OFF $\mathbf{v}$ Submit \\ Switch CFL 1 ON $\mathbf{r}$ Submit \\ Switch FAN 1 OFF v Submit \\ Switch Tubelight 1 OFF $\mathbf{v}$ Submit}

Figure 5(b): Select the appliance (CFL) to switch it ON

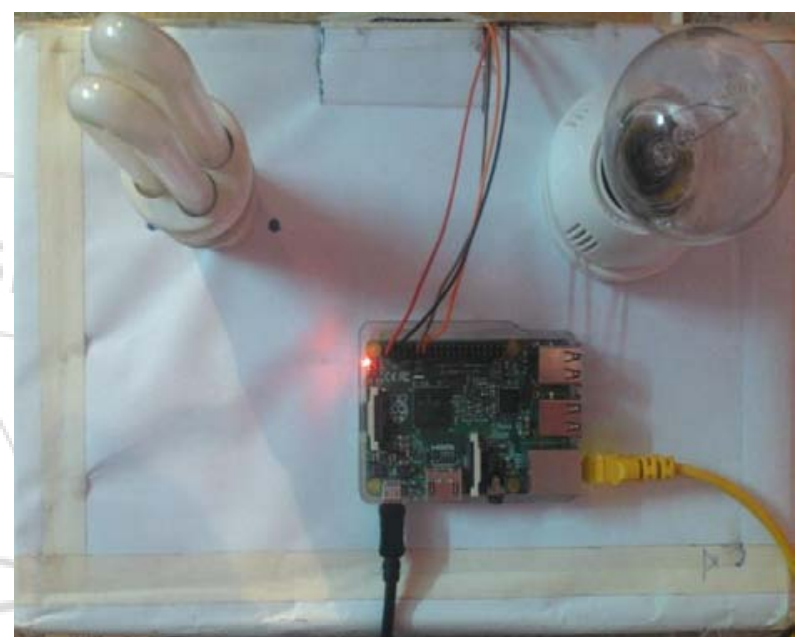

Figure 5(c): A CFL, Bulb interface to RPI

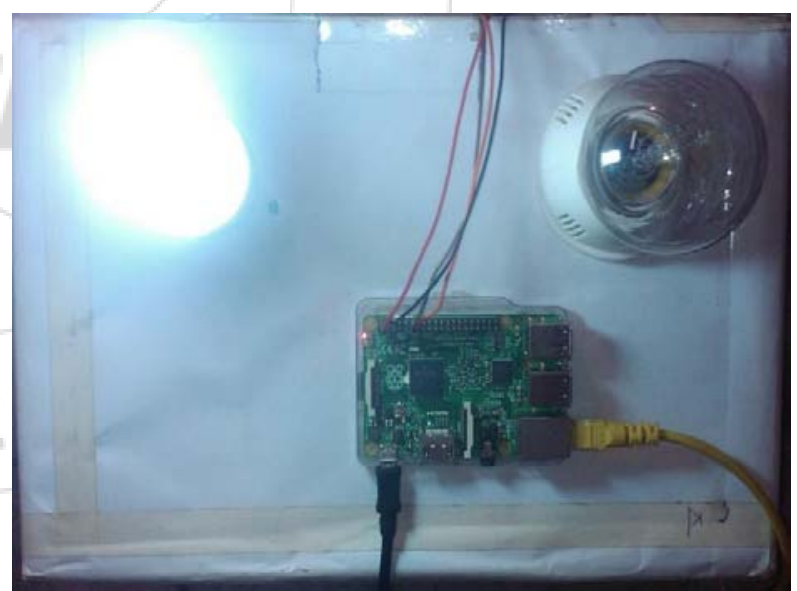

Figure 5(d): Submission of ON from website

\section{Conclusion}

In this Paper the switch control of Electrical appliances through a web interface has been successfully developed. This is proved by the experimental results where in a user had used a web portal to switch ON or OFF a particular device at his house or industry. The paper provides a practical realization of a self-proposed method allowing a safe operating of 23 electrical loads using a single module. Consequently a great deal of research has been done in hardware designing and scripting to address the various problem statements encountered during the process 


\section{Future Improvements}

1. Design of a module which confirms the switching and checks if the electrical appliance is driving power from socket or is faulty.

2. Module to monitor the speed of FAN, AC and light brightness at the house or any place.

3. Embed a live video camera to the microprocessor and send the live feed to the website. Inclusion of CCTV with this module

\section{Acknowledgment}

The author would like to thank Mr. Vuppala Siddhartha, Department of Computer Science and Engineering at MVSREC, Nadergul Hyderabad, India for his relentless and vivacious support in computer programming. And thanks to Dr D.V.M Chary, Head of Department of Electrical and Electronics Engineering at MVSREC for his support and encouragement.

\section{References}

[1] "Unix Shell Programming", Yashavant P. Kanetkar BPB Publications.

[2] "Electronic Devices and Circuits " by J.B Gupta .

[3] Learn Python in 24 hours, by Sam.

[4] Online web Tutorials from www.w3schools.com/

[5] Documentation of Raspberry Pi from https://www.raspberrypi.org/documentation

\section{Author Profile}

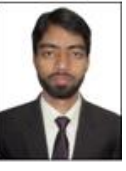

Siraj Ahmed currently a final year B.E student Of Electrical and Electronics Engineering at MVSR Engineering College affiliated to Osmania University , he will be completing his undergraduate degree by June 2016 and is highly focused on research work and a competent academic career .He has done notable interdisciplinary projects relating to electric driven vehicles, embedded systems.

Mir Mohammed Ali is a senior year under graduate student of Electronics and Communication Engineering at Mahaveer Institute of Science and Technology, Bandlaguda, Hyderabad . He is currently working in the research area of Digital processing and Embedded systems.

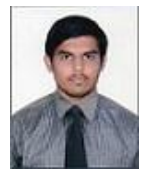

Mohammed Abdul Sami Siddiqui currently pursuing final year of B.Tech in Electrical and Electronics Engineering at Goka Raju Ranga Raju Institute Of Engineering and Technology, Hyderabad .He is keen on exploring different micro controllers and micro processors. 